\title{
A SIMPLE PROOF OF THE FUNDAMENTAL CAUCHY-GOURSAT THEOREM*
}

\author{
BY
}

\author{
ELIAKIM HASTINGS MOORE
}

\section{Introduction.}

Goyrsat in two memoirs (Acta Mathematica, vol. 4, 1884 ; Transactions of the American Mathematical Society, vol. 1, 1900) has proved CAUCHY's integral theorem :

$$
\int_{C} f(z) d z=0
$$

without the assumption of the continuity of the derivative $f^{\prime}(z)$ on the closed region $R$ bounded by the curve of integration $C$, and thereby he has laid deeper foundations for the CAUCHY-RIEmann theory of functions of the complex variable. An abstract of these memoirs is to be found in the Bulletin of this Society for June, 1899, pp. 427-429.

Goursat set out by a direct process $\dagger$ to evaluate the integral in question. In the present paper, by an indirect process, I prove that the integral has the value 0 . The essential elements of the proof are those of Goursat's first paper ; by the modification indicated, and by the imposition on the curve $C$ of a certain condition fulfilled by all the usual curves, one avoids the necessity of introducing the lemma to which Goursat's second paper is devoted.

The necessary preliminary definitions and theorems are given in some detail in $\S 1$, in which connection I refer especially to JoRDan's Cours d'Analyse, $2 d$ ed., vol. 1, 1893, and to Hurwitz's address at the Zurich Congress of 1897 entitled:: Über die Entwickelung der allgemeinen Theorie der analytischen Functionen in neuerer Zeit (Verhandlungen des .. Mathematiker Kongresses in.Zürich ...; Teubner, 1898). Then in $\S \S 2$ and 3 I state and prove the two

\footnotetext{
* Presented to the Society June 29, 1900, at the New York meeting. Received for publication October 30, 1900.

†Stolz (Grundzüge der Differential- und Integralrechnung, vol. 2, p. 218, 1896) states that this process of GouRSAT's first memoir was an improvement of an older method; his reference to LAurent, Théorie_des résidus, 1895, p. 38, I have not succeeded in utilizing.
} 
principal CAUCHY-Goursat theorems corresponding to the two principal forms * of CAUCHY's theorem.

\section{§1. Preliminary definitions and theorems.}

$1^{\circ}$. The following notations are useful in abbreviating general statements involving the notion of limits. With respect to a set $S$ of points in the plane of the complex variable $z$ and a point $z=a$ of the plane, the notation $S_{a}$ indicates the set $S$ with the exception of the point $a$ if $a$ is of the set $S$, and the notation $S_{a \eta}$, where $\eta$ is a positive real number, denotes the subset of $S_{a}$ lying within the circle $(a, \eta)$ of center $a$ and radius $\eta$. The notations may be read thus: the set $S a$ apart; the set $S a$ apart within the $\eta$-neighborhood of $a$.

$2^{\circ}$. The point $z=a$ is a limit point of the set $S$ if every set $S_{a \eta}$ contains one point and so an infinitude of points. The set is closed, if it contains all its limit points.

If a set $S$ is closed and contains an infinite sequence of subsets $S_{\nu}$, each subset $S_{\nu}$ containing an infinitude of points of the set $S$ and containing also the succeeding subset $S_{\nu+1}$, then the subsets $S_{\nu}$ have at least one point of $S$ in common; and if further the $S_{\nu}$ in any way lie respectively in squares forming a sequence of squares with indefinitely decreasing area, then the $S_{\nu}$ have only one point in common.

$3^{\circ}$. A (real or complex) function $f(z)$ is uniquely defined for every point $z$ of a set $S$ with a limit point $z=a$.

The function $f(z)$ has on the $z$-set $S$ for $z=a$ the limit $f$, in symbols,

$$
\underset{\substack{z \backslash S \\ z=a}}{\mathbf{L}} f(z)=f
$$

if for every positive number $\epsilon$ there exists a positive number $\delta_{\epsilon}$ such that

$$
|f(z)-f|<\epsilon \quad\left(z \mid S_{a} \delta_{\epsilon}\right),
$$

for every point $z$ of the set $S_{a \delta_{\epsilon}}$.

The point $z=a$ being of the set $S$, at $z=a$ on the set $S$ the function $f(z)$ is continuous if

$$
\underset{\substack{z \leq S \\ z=a}}{\mathbf{L}_{S}} f(z)=f(a)
$$

and it has the (finite) derivative $f^{\prime}(a)$ if

* Reference should also be made to the following expositions of Cauchy's theorem :

Pringsheim : Münchner Sitzungsberichte, vol. 25, pp. 39-72, 295-304, 1895 ;

Bôcher: Bulletin of the American Mathematical Society, ser. 2, vol. 2, pp. 146-149, 1896 ;

STöLz : loc. cit., p. 217 ff., 1896.

The papers of Pringsheim contain interesting historical data concerning the theorem. 


$$
\underset{\substack{z \mid S \\ z=a}}{\mathbf{L}} \frac{f(z)-f(a)}{z-a}=f^{\prime}(a)
$$

that is, if for the function $\Delta(z)$ defined on the set $S$ by the identity :

$$
f(z)=f(a)+(z-a) f^{\prime}(a)+\Delta(z),
$$

there is for every $\epsilon \mathbf{a} \delta_{\epsilon}$ such that

$$
|\Delta(z)|<\epsilon|z-a| \quad\left(z|S,| z-a \mid<\delta_{\epsilon}\right)
$$

for all points $z$ of the set $S$ within the $\delta_{\epsilon}$-neighborhood of $a$.

$4^{\circ}$. An equation : $z=F(t)$, where $F(t)$ is a complex single valued function of the real variable $t$ on the interval $t_{0} \cdots T$, defines in the $z$-plane a curve $C$, - a path * of the point $z$ from $z_{0}=F\left(t_{0}\right)$ to $Z=F(T)$.

The curve $C$ is continuous when the function $F(t)$ is continuous.

The arc $z_{0} Z$ of the curve $C$ has as length $\dagger$ the limit, if uniquely existent, of the length of the broken line $z_{0} z_{1} z_{2} \cdots z_{n} Z$ whose vertices correspond to the $n+2$ points $t_{0} t_{1} t_{2} \cdots t_{n} T$ of a partition of the interval $t_{0} T$ into $n+1$ subintervals-the limit as the lengths of all subintervals indefinitely decrease with the indefinite increase of $n$. If the arc $z_{0} Z$ has a length, so does its every arc-part.

A rectifiable curve is a continuous curve with length.

It is easy to see in how far the path-curve in definition and properties is inde pendent of the particular parameter $t$.

$5^{\circ}$. The definite integral of a function $f(z)$ defined upon a path $C$ along that path has the definition :

$$
\int_{C} f(z) d z=\coprod_{\delta_{\kappa}=0} \sum_{\kappa=0}^{n} f\left(\zeta_{\kappa}\right)\left(z_{\kappa+1}-z_{\kappa}\right)
$$

where $z_{n+1}$ is $Z$, and $\zeta_{\kappa}$ is any point on the arc $z_{\kappa} z_{\kappa+1}$, and $\delta_{\kappa}$ is $t_{\kappa+1}-t_{\kappa}$.

This $\ddagger$ definite integral exists if the path $C$ is rectifiable and the function $f(z)$ is continuous on $C$ (JORDAN : loc. cit., $\$ \S 193-196,1893)$.

* For present purposes we need to consider only paths lying entirely in the finite plane and containing their extreme points.

† ScHEEFER: Acta Mathematica, vol. 5, pp. 49-82, 1884 ; Ascoli (Cf. Jahrbuch der Mathematik, vol. 16, p. 339, [1884]) ; JoRDAN : loc. cit., $8 \&$ 105-113, 1893 ; STUDY : Mathematische Annalen, vol. 47, pp. 298-316, 1896.

$\ddagger$ The two real or complex functions $P(z), Q(z)$ being defined upon a path $C$, the definite integral of the binomial differential expression $P(z) d x+Q(z) d y$ has the similar definition :

$$
\int_{C} P(z) d x+Q(z) d y=\mathbf{L}_{\delta_{\kappa}=0} \sum_{\kappa=0}^{n} P\left(\zeta_{\kappa}\right)\left(x_{\kappa+1}-x_{\kappa}\right)+\mathbf{L}_{\delta_{\kappa}=0} \sum_{\kappa=0}^{n} Q\left(\zeta_{\kappa}\right)\left(y_{\kappa+1}-y_{\kappa}\right) .
$$

By a proof similar to that cited above one has the theorem : this definite integral exists if the path $C$ is rectifiable and the functions $P(z), Q(z)$ are continuous on $C$. I take occasion to refer to this existence theorem since it seems to be neither in JoRDAN's Cours d'Analyse nor in PICARD's Traité d'Analyse, and since PRINGSheIm (loc. cit., pp. 48-55, 1895) carefully expounds for a certain class of curves a definition and an existence theorem which are in fact special cases of the definition and theorem just given. Pringsheim seems to be unfortunately out of touch with the current notion of the general rectifiable curves (Cf. pp. $48,49,55,59,60$ ). 
$6^{\circ}$. Of a set $S$ a point $a$ of the plane is $(\alpha)$ an inner point, $(\beta)$ an outer point, or $(\gamma)$ a boundary point, according as $(a, \beta)$ there exists a neighborhood of $a,(\alpha)$ all of whose points belong to $S,(\beta)$ none of whose points belong to $S$, or $(\gamma)$ every neighborhood of $a$ contains at least one point of $S$ and one point not of $S$.

A set $S$ is connected if any two of its points $z_{0} Z$ may be joined by a broken line $z_{0} z_{1} \cdots z_{n} Z$ of a finite number of links whose extremities $z_{1} \cdots z_{n}$ are inner points of $S$ and each of length less than a preassigned length.

A set connected and made up exclusively of inner points is with Hurwitz called a continuum.

$7^{\circ}$. A simply* closed continuous curve is a continuous curve whose extreme points coincide but whose points are otherwise distinct. These curves have been carefully studied by JoRDAN (loc. cit., $\S \S 96-113$ ).

Such a curve serves as the boundary between two regions of the plane, the exterior region and the interior region. These regions are continua; after the addition of the curve each region remains connected.

$8^{\circ}$. A continuum is simply connected if every simply closed continuous curve lying in the continuum has its interior region lying entirely in the continuum. Every continuous curve lying in a simply connected continuum lies in the interior of a simply closed continuous curve lying in the same continuum. One readily constructs such a curve in connection with a sufficiently fine grating of squares superposed on the continuum.

\section{§ 2. The Cauchy-Goursat theorem}

for integrals over the boundary of simply connected regions.

\section{Theorem. The definite integral}

$$
\int_{C} f(z) d z
$$

exists and has the value 0 , if

1) the path of integration $C$ is a simply closed continuous rectifiable curve met by the various lines parallel to the xy axes in the z-plane $(z=x+i y)$ in a finite number of points and segments of coincidence, and moreover having the property 2);

$2) \dagger$ for every point $\zeta$ of $C$, if a square with sides parallel to the axes converges in any way to the point $\zeta$, the ratio of the total length of the arcs of $C$

* HuRwitz's "simply" is preferable to JoRDAN's "without multiple point."

$\dagger$ As indicated in the introduction the theorem with the omission of this condition on the curve of integration remains true. It would then be interesting to inquire whether this property of the curve follows from those of hypothesis 1). The usual curves of integration obviously satisfy the condition 2) for the value $\rho_{\zeta}=1$. In particular, the proof here given is sufficient to extend the CAUCHY theorem as given by Jordan to the corresponding CaUCHY-Goursat form (Cf. \& 3 ). 
lying on the square to the perimeter of the square is ultimately less than a certain constant $\rho_{\zeta}$, which may vary as $\zeta$ traverses $C$;

3) on the region $R$, consisting of the curve $C$ and its interior region, the integrand function $f(z)$ is a single valued continuous function of $z$ with a single valued derivative $f^{\prime}(z)$.

In the first place, the integral

exists $\left(\S 1,5^{\circ}\right)$.

$$
J=\int_{C} f(z) d z
$$

We are to prove then that $J=0$. The proof * is indirect. We set

and suppose that $\eta_{0}>0$.

$$
J=J_{0}, \quad\left|J_{0}\right|=\eta_{0},
$$

The region $R=R_{0}$ lies within some square $S_{0}$ with sides parallel to the $x y$ axes and of length $\gamma_{0}$. By the introduction of two diameters we subdivide this square $S_{0}$ into four equal squares $S_{1}^{\prime}, S_{1}^{\prime \prime}, S_{1}^{\prime \prime \prime}, S_{1}^{\text {iv }}$. In view of hypothesis 1) we admit the usual dissection arguments. The region $R$ is subdivided into a finite number of regions bounded by simply closed continuous curves pieced together from pieces of $C_{0}$ and of the two diameters of $S_{0}$. These regions lie each in one of the smaller squares. We denote for brevity the lot of regions in $S_{1}^{\prime}$ by $R_{1}^{\prime}$, and by $C_{1}^{\prime}$ the lot of bounding curves described each in the sense dictated by the sense of description of $C_{0}$, and by

$$
J_{1}^{\prime}=\int_{c_{1}^{\prime}} f(z) d z \quad\left(\left|J_{1}^{\prime}\right|=\eta_{1}^{\prime}\right)
$$

the sum of the integrals of $f(z) d z$ over those path-curves, and so for $S_{1}^{\prime \prime}, S_{1}^{\prime \prime \prime}, S_{1}^{\text {iv }}$.

Then we have

$$
J_{0}=J_{1}^{\prime}+J_{1}^{\prime \prime}+J_{1}^{\prime \prime \prime}+J_{1}^{\mathrm{iv}}, \quad 0<\eta_{0} \leqq \eta_{1}^{\prime}+\eta_{1}^{\prime \prime}+\eta_{1}^{\prime \prime \prime}+\eta_{1}^{\mathrm{iv}} .
$$

Hence one of the four $\eta_{1}$ 's must be $\geqq \eta_{0} / 4$. We denote this $\dagger \eta_{1}$ by the notation $\eta_{1}$ without superscript, and so for the corresponding $J_{1}, C_{1}, R_{1}, S_{1}$. Hence in the square $S_{1}$ we have a state of affairs similar to that initially existing in the square $S_{0}$, except that $R_{1}$ consists of several regions of the type $R_{0}$ for which we are considering the sum of the corresponding integrals each in the sense specified,-a fact however which does not interfere with the indefinite repetition of the dissection process.

\footnotetext{
* The proof is in spirit indirect, although it may immediately be cast in the direct form,-a fact remarked by my colleague Mr. MASCHKE.

$\dagger$ In case of ambiguity the choice is a matter of indifference.
} 
Thus for every integer $\nu$ there is a square $S_{\nu}$ of length $\gamma_{0} / 2^{\nu} ; S_{\nu}$ contains $R_{\nu}$ consisting of a finite number of regions, bounded each by a simply closed continuous curve; these curves each described in the proper sense form the complete boundary $C_{\nu}$ of $R_{v}$; the integral

$$
J_{\nu}=\int_{C_{\nu}} f(z) d z
$$

is the sum of the integrals of $f(z) d z$ over these curves so described; further,

$$
\left|J_{\nu}\right|=\eta_{\nu} \geqq \frac{\eta_{0}}{4^{\nu}}
$$

This dissection process determines a definite point $z=\zeta$ which lies on every square $S_{\nu}(\nu=0,1,2, \cdots)$. It is therefore a limit point of the region $R$, - since every $R_{\nu}$ contains in fact a (simple or compound) curve of integration $C_{\nu}$, - and it is, indeed, since $R$ is closed, a point of $R$, either an inner point or a point of the boundary $C$. For every point $z$ of the square $S_{\nu}$ one has

$$
|z-\zeta| \leqq 2 \gamma_{0} 2^{-\nu}
$$

We set for points $z$ of the region $R$

$$
f(z)=f(\zeta)+(z-\zeta) f^{\prime}(\zeta)+\Delta(z) .
$$

Introducing a positive number $\epsilon$ subject to later determination we have, by hypothesis 3 ) in accordance with $3^{\circ}$ of $\S 1$, a positive number $\delta_{\epsilon}$ such that on the region $R$ within the circle $\left(\zeta, \delta_{\epsilon}\right)$

$$
|\Delta(z)|<\epsilon|z-\zeta| \quad\left(z|R ;| z-\zeta \mid<\delta_{\varepsilon}\right) .
$$

Choosing $\nu_{\epsilon}$ so that the square $S_{\nu_{\epsilon}}$ lies within the circle $\left(\zeta, \delta_{\epsilon}\right)$, e. g., so that

$$
2 \gamma_{0} 2^{-\nu_{\epsilon}}<\delta_{\epsilon},
$$

we have on every region $R_{\nu}\left(\nu \geqq \nu_{\epsilon}\right)$

$$
|\Delta(z)|<\epsilon|z-\zeta| \leqq 2 \epsilon \gamma_{0} 2^{-\nu} \quad\left(z \mid R_{\nu}, \nu \geqq v_{\varepsilon}\right) .
$$

Then on integrating the trinomial expression for $f(z) d z$ along the various path-curves making up $C_{\nu}\left(\dot{\nu} \geqq \nu_{\epsilon}\right)$, remembering that over such closed curves $\int d z=0, \int z d z=0$, and applying the usual mean value theorem, we have

$$
J_{\nu}=\int_{C_{\nu}} \Delta(z) d z ; \quad\left|J_{\nu}\right|=\eta_{\nu}<2 \epsilon \gamma_{0} 2^{-\nu} \lambda_{\nu}^{\prime} \quad\left(\nu \geqq v_{\varepsilon}\right),
$$

where $\lambda_{\nu}^{\prime}$ is the total length of the curves of $C_{\nu}$. Then

$$
\lambda_{\nu}^{\prime} \leqq 4 \gamma_{0} 2^{-\nu}+\lambda_{\nu},
$$


where $\lambda_{\nu}$ is the total length of the arcs of the original curve $C$ on the square $S_{\nu}$ of perimeter $4 \gamma_{0} 2^{-\nu}$. Hence, by hypothesis 2), for all $\nu$ 's greater than a certain* $\nu_{0}$

$$
\lambda_{\nu}<4 \gamma_{0} 2^{-\nu} \rho
$$

where $\rho$ is a certain positive constant. Thus

while

$$
\eta_{\nu}<8 \epsilon \gamma_{0}^{2} 4^{-\nu}(1+\rho) \quad\left(\nu \geqq v_{\epsilon} ; \nu>v_{0}\right),
$$

$$
\eta_{\nu} \geqq \eta_{0} 4^{-\nu} \quad(v=0,1,2, \cdots) .
$$

Thus by the mediation of a properly chosen $\nu$ one has the relation

$$
8 \epsilon \gamma_{0}^{2}(1+\rho)>\eta_{0}
$$

which is independent of $\nu$. But the positive number $\epsilon$ remains at our disposal and may now be chosen so that

$$
8 \epsilon \gamma_{0}^{2}(1+\rho)<\eta_{0},
$$

since $\eta_{0}>0$. Thus we reach the contradiction needed to complete the proof of the theorem.

\section{§3. The Cauchy-Goursat theorem}

for integrals over a closed rectifiable curve lying on a continuum.

We may with Hurwitz (loc. cit., pp. 101-103) formulate the CAUChY theorem as proved by JoRDAN (loc. cit., $\$ § 196-198$ ) as follows :

THEOREM. The definite integral

$$
\int_{C} f(z) d(z)
$$

exists and has the value 0 , if the path of integration $C$ is a closed continuous rectifiable curve lying on a simply connected continuum on which the integrand function $f(z)$ is synectic.

A function $f(z)$ existing as a single valued function of $z$ on a continuum is synectic if for every such $z$ it is continuous and has a derivative in each axial direction, these axial derivatives being equal, and this common derivative-value being itself a continuous function on the continuum, or, what is the same thing, if for every such $z$ it is continuous and has a single valued derivative $f^{\prime}(z)$ itself a continuous function on the continuum.

In JoRDAN's proof the theorem is shown to depend upon the special case for a triangle lying on the simply connected continuum. In this reduction the integrand function $f(z)$ enters only with its property of continuity.

\footnotetext{
* If $\zeta$ is an inner point of $R$, then the $R_{\nu}$ ultimately lie within $R$ and the $\lambda_{\nu}$ are ultimately 0 .
} 
Accordingly by the use of the Goursat proof or that of $\S 2$ for this case of the triangle we may enunciate the theorem referred to in the caption of $\S 3$.

Theorem. The definite integral

$$
\int_{C} f(z) d(z)
$$

exists and has the value 0 , if the path of integration $C$ is any closed continuous rectifiable curve lying on a simply connected continuum on which the integrand function $f(z)$ is a single valued continuous function of $z$ with $a$ single valued derivative $f^{\prime}(z)$.

The University of Chicago. 